\title{
Beta-bloqueantes como antihipertensivos de primera línea: la edad del paciente es lo que importa
}

Beta-blockers for the treatment of hypertension: patient's age does matters

Khan N y col. CMAJ 2006; 174:1737-1742.

\section{Objetivo}

Comparar la eficacia de los beta-bloqueantes como fármacos de primera línea para prevenir eventos cardiovasculares mayores en hipertensos jóvenes y añosos.

\section{Fuente y selección de datos}

Los autores analizaron ensayos clínicos controlados y aleatorizados publicados entre 1950 y 2006, que hubieran evaluado la eficacia de los betabloqueantes para prevenir eventos cardiovasculares mayores como terapia de primera línea de la hipertensión.

\section{Resultados principales}

Comparado con placebo, la incidencia de eventos compuestos -muerte, accidente cerebrovascular (ACV) o infarto de miocadio (IAM)- fue menor en los menores de 60 años tratados con betabloqueantes pero no en los mayores de 60 años. Comparados con otros antihipertensivos, los betabloqueantes no mostraron diferencias en la tasa de eventos compuestos en menores de 60 años, pero se asociaron a un exceso de eventos en los pacientes mayores. Ver tabla 1.
Tabla 1: eficacia de los beta bloqueantes en las diferentes subpoblaciones.

\begin{tabular}{l|c|c|c}
\multirow{2}{*}{$\begin{array}{c}\text { Comparación de beta-bloqueantes vs. } \\
\text { placebo y vs. otros anti-hipertensivos }\end{array}$} & \multicolumn{2}{|c}{ Muerte, ACV o IAM } \\
\cline { 2 - 4 } Menores de 60 años & Placebo & RR & CL95\% \\
\cline { 2 - 4 } & Otros anti-hipertensivos & 0,86 & 0,74 a 0,99 \\
\hline \multirow{2}{*}{ Mayores de 60 años } & Placebo & 0,75 a 1,05 \\
\cline { 2 - 4 } & Otros anti-hipertensivos & 1,06 & 0,75 a 1,05 \\
\hline
\end{tabular}

ACV: accidente cerebrovascular. IAM: infarto agudo de miocardio.

En los mayores de 60 años, el uso de betabloqueantes se asoció a mayor riesgo de ACV (RR 1,18; IC95\% 1,07 a 1,30) pero similar incidencia de IAM, insuficiencia cardíaca o muerte.

\section{Conclusiones}

Usados como terapia antihipertensiva de primera línea en menores de 60 años, los betabloqueantes son superiores al placebo y comparables a otros antihipertensivos en la prevención de eventos cardiovasculares mayores. En cambio, en mayores de 60 años, los betabloqueantes no fueron más efectivos que el placebo, y, en comparación con otros antihipertensivos, se asociaron a mayor riesgo de ACV.

Palabras clave: hipertensión arterial, beta bloqueantes, edad.

Key words: hypertension, beta blockers, age.

Fuente de financiamiento: Canadian Institutes of Health Research, Heart and Stroke Foundation of Canada, St. Paul's Hospital Foundation Research, Alberta Heritage Foundation for Medical Research, University of Alberta/Merck Frosst/Aventis.

\section{Comentario}

En los últimos años se ha cuestionado la efectividad de los betabloqueantes como tratamiento de primera línea ${ }^{1}$ del de la hipertensión arterial no complicada, vale decir pacientes sin cardiopatía isquémica o insuficiencia cardíaca; o que no sean candidatos al tratamiento con estos fármacos por otras comorbilidades como migraña, temblor esencial, etc.

Este cuestionamiento está basado en los resultados de distintos metaanálisis que muestran la inferioridad de los betabloqueantes -mayormente atenolol- para prevenir eventos cardiovasculares, en especial ACV en ancianos.

El presente metaanálisis muestra que la inferioridad de los betabloqueantes frente a otros antihipertensivos previamente descripta en ancianos no es extensiva a los pacientes menores de 60 años. En los "jóvenes" se observó superioridad de los betabloqueantes frente al placebo en la prevención de eventos cadiovasculares mayores, y su equivalencia frente a otros antihipertensivos.

\section{Conclusiones del comentador}

Se confirma que el uso de betabloqueantes como terapia de inicio en hipertensos ancianos se asocia a un exceso de accidentes cerebovasculares. Posibles razones explicarían esta falta de beneficio terapéutico: una menor eficacia antihipertensiva, mayor incidencia de diabetes y dislipemia, ineficacia para revertir el remodelado ventricular y microvascular y deterioro del flujo sanguíneo tisular. Queda por demostrar si la menor efectividad terapéutica de los betabloqueantes es un fenómeno de clase o si se limita al atenolol, y si es o no extensible a los nuevos betabloqueantes vasodilatadores como el carvedilol y el nebivolol.

José Alfie [ Sección Hipertensión Arterial del Servicio de Clínica Médica del Hospital Italiano de Buenos Aires jose.alfie@hospitalitaliano.org.ar ]

Alfie J. Beta-bloqueantes como antihipertensivos de primera línea: la edad del paciente es lo que importa. Evid. actual. práct. ambul; 11(4): 103, JulAgo.2008. Khan N, y col. Re-examining the efficacy of b-blockers for the treatmemnt of hypertension: a meta-analysis. CMAJ 2006; 174:17371742. PMID: 16754904. Disponible en URL: http://www.cmaj.ca/cgi/reprint/174/12/1737 (último acceso 25/07/08).

\section{Referencia}

1. National Collaborating Centre for Chronic Conditions.Hypertension: management of hypertension in adults in primary care: partial update. London: Royal College of Physicians, 2006. Disponible en Internet: http://www.nice.org.uk/guidance/CG34 (último acceso 25/07/08). 